\title{
HS 2333+3927: A new sdB+dM binary with a large reflection effect ${ }^{\star}$
}

\author{
U. Heber ${ }^{1}$, H. Drechsel ${ }^{1}$, R. Østensen ${ }^{2}$, C. Karl $^{1}$, R. Napiwotzki ${ }^{1}$, M. Altmann ${ }^{1}$, \\ O. Cordes ${ }^{3}$, J.-E. Solheim ${ }^{4}$, B. Voss ${ }^{5}$, D. Koester ${ }^{5}$, and S. Folkes ${ }^{2}$
}

${ }^{1}$ Dr. Remeis-Sternwarte Bamberg, Astronomisches Institut der Universität Erlangen-Nürnberg, Sternwartstraße 7, 96049 Bamberg, Germany

e-mail: [drechsel;karl;napiwotzki;altmann]@sternwarte.uni-erlangen.de

2 Isaac Newton Group of Telescopes, 37800 Santa Cruz de La Palma, Canary Islands, Spain e-mail: roy@ing.iac.es

3 Sternwarte der Universität Bonn, Auf dem Hügel 71, 53121 Bonn, Germany e-mail: cordes@astro.uni-bonn.de

${ }^{4}$ University of Troms $\varnothing$, Department of Physics, 9037 Troms $\varnothing$, Norway e-mail: janerik@phys.uit.no

5 Institut für Theoretische Physik und Astrophysik, Universität Kiel, 24098 Kiel, Germany e-mail: [koester/voss]@astrophysik.uni-kiel.de

Received 15 January 2004 / Accepted 29 February 2004

\begin{abstract}
We have discovered periodic light variations $(P=0.1718023 \mathrm{~d})$ in the sdB star HS $2333+3927$ in the $B V R$ bands with amplitudes of $0.21,0.28$ and $0.33 \mathrm{mag}$, respectively. Sinusoidal radial velocity variations at the same period were detected with a semi-amplitude of $K_{1}=89.6 \mathrm{~km} \mathrm{~s}^{-1}$, indicating that it is binary system and that the light variations are caused by the reflection effect with no eclipses. A mass function of $f(m)=0.0128 M_{\odot}$ has been determined. The analysis of the light curve did not yield a unique solution, mainly because the albedo of the secondary is poorly constrained. Two solutions of equal quality with a high $\left(A_{2}=1.0\right)$ and a low $\left(A_{2}=0.39\right)$ albedo were considered further. Variability of the Balmer line profiles, most notably for $\mathrm{H} \alpha$, was discovered, probably also caused by the reflection effect. A spectroscopic analysis results in $T_{\text {eff }}=36500 \mathrm{~K}, \log g=5.70$, and $\log \left(n_{\mathrm{He}} / n_{\mathrm{H}}\right)=-2.15$. These characteristics are typical for sdB stars. Mass-radius relations are derived from the results of the analysis of light and radial-velocity curves. Comparison with the observed mass-radius relation of the sdB star and with that of lower main sequence stars for the companion allows us to discard the high albedo solution, because the resulting mass of the primary and the radius of the secondary would be unreasonably low. From a discussion of evolutionary models we constrain the plausible mass of the sdB to the range between $0.29 M_{\odot}$ and $0.47 M_{\odot}$. Accordingly, the mass of the secondary is between $0.24 M_{\odot}$ and $0.32 M_{\odot}$, indicating a spectral type of M3 to M4. HS $2333+3927$ is only the sixth sdB $+\mathrm{dM}$ system discovered so far. An improved measurement of the gravity and the projected rotational velocity of the sdB star is required to further constrain the masses and to identify the evolutionary state of the sdB star uniquely.
\end{abstract}

Key words. stars: subdwarfs - stars: binaries: spectroscopic - stars: binaries: close - stars: early-type stars: fundamental parameters - stars: individual: HS 2333+3927

\section{Introduction}

Subluminous B ( $\mathrm{sdB}$ ) stars dominate the populations of faint blue stars of our own Galaxy and are found in both the old disk and in halo populations, e.g. as stars forming the blue tails

Send offprint requests to: $\mathrm{U}$. Heber,

e-mail: heber@sternwarte.uni-erlangen.de

* Based on observations obtained at the German-Spanish Astronomical Center (DSAZ) at Calar Alto, the Nordic Optical Telescope (NOT) and the Jacobus Kapteyn Telescope (JKT) on La Palma, the Hoher List Observatory in Germany and the $80 \mathrm{~cm}$ telescope of the Instituto de Astrofisica de Canarias (IAC80) on Tenerife. to the horizontal branches of globular clusters (Ferraro et al. 1997).

However, important questions remain over the evolutionary paths and the appropriate time scales. There is general consensus that the sdB stars can be identified with models for Extreme Horizontal Branch (EHB) stars (Heber 1986; Saffer et al. 1994). Like all HB stars they are core helium burning objects. However, their internal structure differs from typical HB stars, because their hydrogen envelope is very thin $(<1 \%$ by mass) and therefore inert. As a consequence EHB stars evolve directly to the white dwarf cooling sequence, thus avoiding a second red giant phase (Dorman et al. 1993). 
The discovery of multi-mode, short-period $(P=2-10 \mathrm{~min})$ pulsators among sdB stars (see O'Donoghue et al. 1999 and Charpinet 2001 for reviews) has opened a new attractive possibility of probing their interiors via seismological tools. Recently, Brassard et al. (2001) were able to derive the mass and hydrogen envelope mass of the pulsating sdB star PG $0014+067$ by asteroseismology. Their results (total mass $M=0.49 \pm 0.019 M_{\odot}$ and envelope mass $\log \left(M_{\text {env }} / M_{\odot}\right)=$ $-4.3 \pm 0.22)$ are in excellent agreement with the predictions by evolution theory.

Considerable evidence is accumulating that many sdB stars reside in close binaries (Maxted et al. 2001; Saffer et al. 2001; Napiwotzki et al. 2004). Therefore mass transfer should play an important role in the evolution of such binary systems. Detailed investigations of sdB binaries, in particular eclipsing systems, are crucial to determine their masses. However, only three such eclipsing binaries, HW Vir (Menzies \& Marang 1986), PG 1336-018 (Kilkenny et al. 1998), and HS 0705+6700 (Drechsel et al. 2001) are known up to now, which consist of an sdB star and an optically invisible M dwarf companion. Here we report the discovery of another related system HS 2333+3927, which is, however, not eclipsing, but otherwise possesses very similar system parameters and configuration.

HS $2333+3927$ was selected from a list of candidates drawn from the Hamburg Schmidt (HS) survey (Hagen et al. 1995). Follow-up spectroscopy (Heber et al. 1999; Edelmann et al. 2003) revealed that its effective temperature lies in the domain predicted for systems showing pulsational instability. The photometric variability of HS $2333+3927$ was first discovered in a monitoring run of about $2 \mathrm{~h}$ duration at the Nordic Optical Telescope on 19 October 1999, during a search campaign for sdB pulsators (see Østensen et al. 2001a,b). The lightcurve had shown a clear long-term trend, where the target dimmed by about $20 \%$. A discussion of these data is given by Østensen (2000), where a reflection effect was already suggested as the most likely explanation for the photometric variability. Though the star was included in the survey of pulsating northern sdB stars by Billeres et al. (2002), it was not found to be variable there, because it had only been checked for shortterm pulsational variability.

Radial velocity variations of HS $2333+3927$ were detected in two optical spectra (Edelmann et al. 2003), indicating that it is a binary star. Therefore additional photometry was secured in July 2002 at the $1 \mathrm{~m}$ Jacobus Kapteyn Telescope (JKT), La Palma, which confirmed its photometric variability and revealed that the light variations are periodic, but not on a time and flux scale expected for pulsations. Between July and November 2002, CCD photometry was collected at various observatories. In August 2002 the complete radial velocity curve was measured. Section 2 describes the photometric and spectroscopic observations. A photometric solution of the light curve is obtained in Sect. 3. Section 4 describes the spectroscopic analysis (determination of radial velocity curve, discussion of phase-dependent line profile variations and quantitative spectral analysis to derive atmospheric parameters). The evolutionary status of the system is discussed in Sect. 5, and conclusions are drawn in Sect. 6.

\section{Observations}

\subsection{Photometry}

The first photometric observations of HS $2333+3927$ were made in a monitoring run of $5780 \mathrm{~s}$ duration at the Nordic Optical Telescope on 19 October, 1999 (for details on the instrumentation and method see Østensen et al. 2001a). These CCD observations consisted of 289 points of $20 \mathrm{~s}$ integrations in the $B$ filter, taken differentially with respect to three reference field stars. A descending part of the light curve was covered, and the star had probably reached a minimum shortly before setting and termination of the run (which is consistent with the ephemeris derived from our new observations).

Further photometric $B V R$ measurements of HS $2333+3927$ were collected with four different telescopes between July and November 2002. A journal of observations is presented in Table 1. The initial observations were obtained with the $1 \mathrm{~m}$ Jacobus Kapteyn Telescope (JKT) of the Roque de los Muchachos Observatory (La Palma, Canary Islands, Spain) equipped with the JAG-CCD camera and $B$ filter on four nights between 25 and 29 July 2002. $R$ filter measurements were made in one night (17/18 Aug. 2002) with the CCD camera of the $1 \mathrm{~m}$ telescope of the Hoher List Observatory of the University Bonn, Germany. On four nights between 16 and 20 Aug. 2002, the $1.23 \mathrm{~m}$ telescope of the Calar Alto Observatory, Spain, was used to monitor the star with $B$ and $V$ filter CCD photometry. Two more nights (8/9 and 9/10 Oct. 2002) at Hoher List Observatory were dedicated to measure the $V$ light curve again. Finally, in the night of 10/11 Nov. 2002 we obtained another $B$ light curve of the star with the IAC $0.80 \mathrm{~m}$ telescope of Teide Observatory, Tenerife, Spain, equipped with the Troms $\varnothing$ CCD Photometer (TCP), our own portable CCD camera designed for high-speed photometry of variable stars (see Østensen 2000).

Table 1 lists the time of observation (date, start UT), duration of continuous monitoring, number of individual frames, telescope, instrument, filter and observer.

The observations with the JKT telescope were all obtained in the Harris $B$ filter, and the exposure times were $30 \mathrm{~s}$ on the first and last night, and $90 \mathrm{~s}$ on the other two. The dead time between exposures on the $2 \mathrm{k} \times 2 \mathrm{k}$ SITe CCD was about $45 \mathrm{~s}$, kept reasonably low by windowing the CCD with a strip of about $1200 \times 290$ pixels. The data were processed using our own Real Time Photometry (RTP) software, that includes bias level correction from overscan, flatfield correction, and sky level processing, as well as aperture photometry and extinction corrections from multi-windowed CCD frames (see Østensen 2000 for details). Some uncertainty remained in the final calibration of the differential magnitudes due to heavy dust, particularly during the final nights of this run.

On one night (17/18 Aug. 2002) Cousins $R$ photometry and on two nights (8/9 and $9 / 10$ Oct. 2002) Johnson $V$ photometry was obtained using the $1.06 \mathrm{~m}$ telescope of the Hoher List Observatory (University Bonn), near Daun, Germany. The HOLICAM CCD camera was equipped with a dual readout $2048 \times 2048$ pixel Loral CCD chip, of which only the central $512 \times 512$ pixels were used to save readout time. 
Table 1. CCD photometry of HS $2333+3927$.

\begin{tabular}{|c|c|c|c|c|c|c|c|}
\hline Night & Start UT & Duration & Frames & 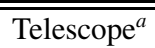 & Instrument & Filter & Observer $^{b}$ \\
\hline 18/19-10-99 & $2: 27: 50$ & $1^{\mathrm{h}} 36^{\mathrm{m}}$ & 289 & NOT & CCD CAM & $B$ & JES, RØ \\
\hline $25 / 26-07-02$ & $3: 49: 10$ & $1^{\mathrm{h}} 54^{\mathrm{m}}$ & 50 & JKT & JAG-CCD & $B$ & $\mathrm{R} \varnothing$ \\
\hline $26 / 27-07-02$ & $2: 51: 12$ & $2^{\mathrm{h}} 45^{\mathrm{m}}$ & 76 & JKT & JAG-CCD & $B$ & $\mathrm{R} \varnothing$ \\
\hline $27 / 28-07-02$ & $1: 27: 55$ & $4^{\mathrm{h}} 14^{\mathrm{m}}$ & 66 & JKT & JAG-CCD & $B$ & $\mathrm{R} \varnothing$ \\
\hline 28/29-07-02 & $1: 31: 13$ & $1^{\mathrm{h}} 21^{\mathrm{m}}$ & 30 & JKT & JAG-CCD & $B$ & SF \\
\hline $16 / 17-08-02$ & $21: 54: 00$ & $6^{\mathrm{h}} 08^{\mathrm{m}}$ & 10 & $\mathrm{CA}$ & CCD cam & $B$ & VK \\
\hline 17/18-08-02 & 21:08:56 & $5^{\mathrm{h}} 04^{\mathrm{m}}$ & 7 & $\mathrm{CA}$ & CCD cam & $B$ & VK \\
\hline 18/19-08-02 & 20:06:09 & $8^{\mathrm{h}} 20^{\mathrm{m}}$ & 14 & $\mathrm{CA}$ & CCD cam & $B$ & VK \\
\hline 19/20-08-02 & $22: 16: 02$ & $5^{\mathrm{h}} 11^{\mathrm{m}}$ & 12 & $\mathrm{CA}$ & CCD cam & $B$ & VK \\
\hline $16 / 17-08-02$ & $22: 00: 20$ & $5^{\mathrm{h}} 58^{\mathrm{m}}$ & 10 & $\mathrm{CA}$ & CCD cam & V & VK \\
\hline 17/18-08-02 & $21: 17: 25$ & $4^{\mathrm{h}} 49^{\mathrm{m}}$ & 7 & $\mathrm{CA}$ & CCD cam & V & VK \\
\hline 18/19-08-02 & 20:00:14 & $8^{\mathrm{h}} 19^{\mathrm{m}}$ & 14 & $\mathrm{CA}$ & CCD cam & V & VK \\
\hline 19/20-08-02 & $22: 30: 35$ & $5^{\mathrm{h}} 05^{\mathrm{m}}$ & 13 & $\mathrm{CA}$ & CCD cam & V & VK \\
\hline 17/18-08-02 & $21: 35: 33$ & $5^{\mathrm{h}} 30^{\mathrm{m}}$ & 181 & HL & CCD cam & $R$ & MA \\
\hline 8/09-10-02 & $19: 35: 43$ & $8^{\mathrm{h}} 19^{\mathrm{m}}$ & 159 & HL & CCD cam & V & $\mathrm{OC}$ \\
\hline $9 / 10-10-02$ & $20: 37: 27$ & $5^{\mathrm{h}} 02^{\mathrm{m}}$ & 115 & $\mathrm{HL}$ & CCD cam & V & OC \\
\hline 10/11-11-02 & $21: 35: 00$ & $4^{\mathrm{h}} 02^{\mathrm{m}}$ & 201 & IAC & TCP FASU & $B$ & JES \\
\hline
\end{tabular}

${ }^{a}$ NOT: 2.5 m Nordic Optical Telescope, La Palma, Canary Islands, Spain; JKT: 1 m Jacobus Kapteyn Telescope, La Palma, Canary Islands, Spain; CA: 1.23 m Calar Alto, Spain; HL: 1 m Hoher List, Bonn, Germany; IAC: 0.80 m telescope, Instituto de Astrofísica de Canarias, Teide Observatory, Tenerife, Canary Islands, Spain.

${ }^{b}$ MA: M. Altmann; OC: O. Cordes; SF: S. Folkes; RØ: R. Østensen; JES: J.-E. Solheim; VK: B. Voss and D. Koester.

The exposure time of each frame was $90 \mathrm{~s}$, adding up with the readout and storage time to a cycle time of approximately $105 \mathrm{~s}$. Basic image reduction (bias subtraction, flatfield corrections) was accomplished with the IRAF data reduction package. Fluxes of the target star and of six reference stars were derived using the package SExtractor (Bertin \& Arnouts 1996). Differential fluxes of HS $2333+3927$ were then determined with respect to the centroid of the reference stars. Since no standard fields had been observed, no transformation into the standard system was made.

On four nights from 16 to 20 August 2002, Johnson $B$ and $V$ photometry was obtained at the Calar Alto Observatory using the $1.23 \mathrm{~m}$ Telescope with a CCD camera equipped with a $2 \mathrm{k} \times$ $2 \mathrm{k}$ SITe chip. The integration times were $180 \mathrm{~s}$ for Johnson $B$ and $90 \mathrm{~s}$ for Johnson $V$ exposures. Both $B$ and $V$ measurements were taken at intervals of typically $30 \mathrm{~min}$, interleaved with other (in part standard star) observations. Observations of standard star fields were used to transform the instrumental magnitudes to the standard system. Four stars from the field around the target were selected as reference objects to derive differential magnitudes. The IRAF package was applied for basic data reduction, aperture photometry and the transformation of magnitudes.

Our last run on HS $2333+3927$ was made on the night of 10/11 November 2002, using the IAC80 telescope and the TCP camera. The TCP camera is equipped with a thinned Tektronix $1024 \times 1024$ CCD chip, and the shutter is hardware synchronized with a GPS receiver for accurate timing. The Johnson $B$ band measurements were made with a cycle time of $60 \mathrm{~s}$, of which $57.4 \mathrm{~s}$ was spent integrating. The low dead-time was achieved by windowing the target, three reference stars and two sky fields down to $50 \times 50$ pixels only, and starting the next exposure immediately after the completion of CCD readout, thus eliminating system overheads for image transfer and storage (see Østensen 2000 for details).

\subsection{Spectroscopy}

HS 2333+3927 was spectroscopically observed during a run at the Calar Alto Observatory of the German-Spanish Astronomical Center, Spain. With the TWIN spectrograph at the $3.5 \mathrm{~m}$ telescope a total of 18 medium resolution spectra were taken from 11 to 18 August 2002. For the blue arm we used grating Nos. 5, and 6 for the red one. Both gratings have 1200 lines/mm, yielding a dispersion of $0.54 \AA$ per pixel. The wavelength coverage ranges from $3900 \AA$ to $5000 \AA$ at a resolution of $1.3 \AA$ in the blue part of the spectrum, and from $6000 \AA$ to $7000 \AA$ at a resolution of $1.2 \AA$ in the red part. A set of dome flatfields and bias calibration frames were taken at the beginning and at the end of each night. In order to check the stability of the instrument over the period of the exposure, a ThAr exposure was taken before and after each scientific exposure in order to ensure an accurate wavelength calibration.

In addition HS 2333+3927 was observed at the Calar Alto Observatory with the CAFOS spectrograph at the $2.2 \mathrm{~m}$ telescope. A total of 13 low resolution spectra were taken on 31 August, 2003. With grism B100 the wavelength range from $3300 \AA$ to $6000 \AA$ was covered at a spectral resolution 


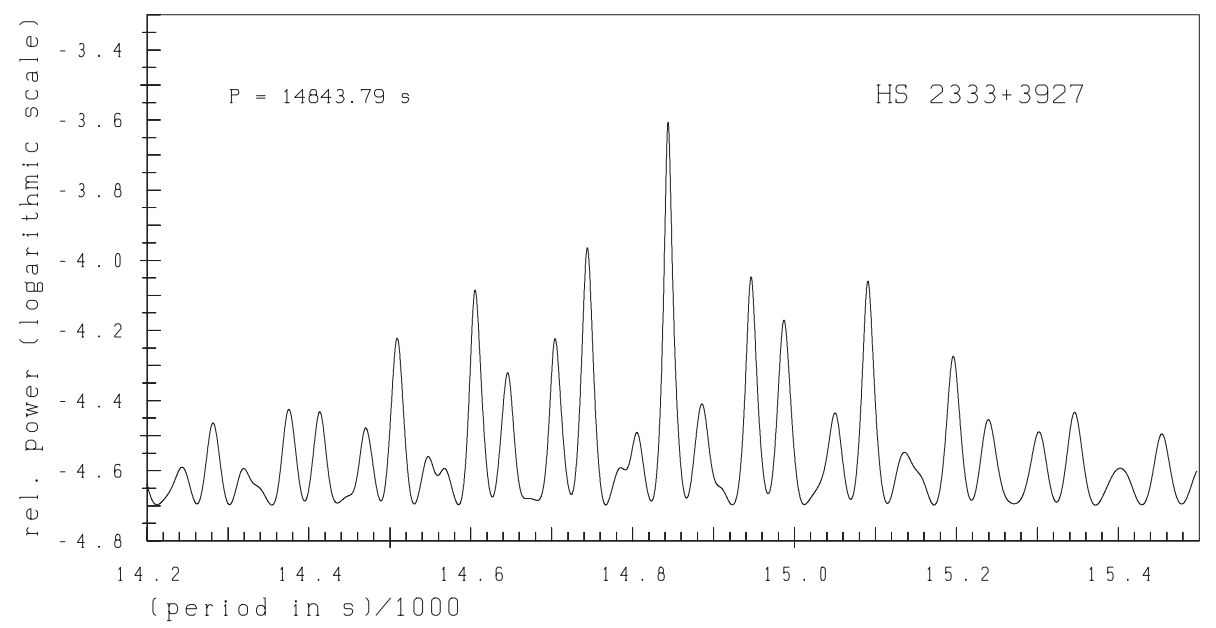

Fig. 1. Power spectrum of HS $2333+3927$ based on $B V R$ photometry of 12 observing nights between 25 July and 10 Nov. 2002 ; total search interval was $1-10 \mathrm{~h}$, highest power was found at $P=14843.72 \pm 0.08 \mathrm{~s}$.

of $4.5 \AA$. This allowed to measure the entire Balmer series (except $\mathrm{H} \alpha$ ) to the Balmer jump.

The data were reduced with a set of standard routines for long slit spectra from the ESO MIDAS package. A 2-dimensional bias correction was applied for the object data as well as for the dome flatfields. After flatfield correction, the spectra were extracted from the 2-dimensional images with respect to the sky background. Finally we performed the wavelength calibration using one of the ThAr exposures (for the TWIN spectra) and the $\mathrm{He}-\mathrm{Hg}$-Cd-Rb lamp exposures (for the CAFOS spectra) attributed to each stellar spectrum.

\section{Photometric analysis}

\subsection{Determination of ephemeris}

The $B$ filter measurements taken in the night 25/26-07-2002 with the JKT telescope covered a full minimum of the sineshaped light curve. Fifty individual exposures with a mean cycle time of about $140 \mathrm{~s}$ were incidentally distributed rather symmetrically around the central part of a minimum. A parabola was fitted to the run of these observations and yielded the reference minimum time of our new ephemeris (after heliocentric correction: 26-07-2002, UT $4^{\mathrm{h}} 50^{\mathrm{m}} 41^{\mathrm{s}}=$ HJD $2452481.7019 \pm 0.0023$ ).

From the overall run of the $B$ light curve covered during the four subsequent observing nights at the JKT it was evident that the period must lie in the range of a few hours, say between 3 and $6 \mathrm{~h}$. To perform a periodogram analysis of the data of 25 to 29-07-2002, the differential photometry of these four nights was relatively calibrated by normalizing the flux level of all nights to that of the reference minimum of the first night, because in each of the observing nights a minimum was fully or at least partly covered. Within a search interval between 1 and $10 \mathrm{~h}$, the highest power was found for $P=14839$ s $(0.17175 \mathrm{~d})$. When phasing the four nights with this preliminary period, one can clearly recognize a reflectiondominated near sinusoidal variability pattern, with no evidence for eclipses.
The period determination could be refined by widening the time base under inclusion of the later $B V R$ observations obtained with CCD cameras at the $1.23 \mathrm{~m}$ Calar Alto $(B V)$ and $1 \mathrm{~m}$ Hoher List telescopes (VR) in August and October 2002, and at the IAC $0.80 \mathrm{~m}$ telescope (B) of Teide Observatory in November 2002. Again, a relative calibration was applied to shift the differential photometric fluxes of the different photometric systems and filters to the same mean flux level. Though the amplitudes of the $B V R$ curves are not the same $(\Delta B=0.21$, $\Delta V=0.28$, and $\Delta R=0.33 \mathrm{mag}$ ), their similar sine-shaped profile still allowed for a common evaluation of this combined data set. The extended time base yielded an improved period of $14843.72 \mathrm{~s}(0.1718023 \mathrm{~d} \pm 0.0000009)$. The power spectrum is shown in Fig. 1.

As a result the following ephemeris was determined, which could be used for a unique phasing of our photometric and spectroscopic observations:

$$
\begin{gathered}
\mathrm{HJD}=2452481 \mathrm{~d} .7019+0.1718023 \times E \\
\pm 23
\end{gathered}
$$

\subsection{Light curve solution}

The CCD photometry obtained in four nights 25-29 July 2002 with the La Palma JKT telescope ( $B$ filter) and with the $1 \mathrm{~m}$ telescope at Bonn Hoher List Observatory (two nights: 8-10 Oct. 2002, $V$ filter and one night: $17-18$ Aug. 2002, $R$ filter) was used for a simultaneous analysis of the $B V R$ light curves. The $B$ and $V$ measurements collected with the $1.23 \mathrm{~m}$ Calar Alto telescope were not included in the light curve solution, because of their much coarser time resolution ( 30-40 min). The numerical solution was performed with the Wilson-Devinney (1971) based light curve program MORO (Drechsel et al. 1995). This code was developed for application to close binaries with hot components, and the underlying model for the binary structure is a modified Roche model taking into account the mutual radiative interaction of the two binary components, which is non-negligible in the 
case of OB-type systems in close configurations. In contrast to the classical Wilson-Devinney model, the SIMPLEX method is used as optimization algorithm. For details on the treatment of radiation pressure effects and properties of the numerical procedure the reader is referred to a general description given by Drechsel et al. (1995).

The times of the $B V R$ measurements were heliocentrically corrected and phased using the ephemeris given in Sect. 3.1. These linear elements provide sufficiently accurate phase values within the time span covered by our $B V R$ observations: due to the absence of eclipses and the relatively shallow shape of the reflection effect light curve minimum, the timing of the reference minimum of 26 July 2002 cannot be given to higher precision than \pm 0.013 phase units. The period is derived with a relative accuracy of $\Delta P / P=5 \times 10^{-6}$, so that the effect of an apparent phase shifting due to a possible error of the period value is restricted to a few times 0.001 phase units during the whole covered time interval of observations of 3-4 months. Given the smooth run of light variability, such small error in phase would hardly affect the photometric solution. Hence the ephemeris appears entirely adequate to ensure a consistent phasing when combining the time separated $B V R$ measurements for a simultaneous light curve solution.

Due to the absence of geometrical eclipses and the still large total number of 16 free parameters, a simultaneous solution of the $B V R$ light curves was not a trivial task. A large number of trial runs was necessary to confine the parameters to a physically reasonable range and to find consistent parameter combinations. Trial runs of the SIMPLEX optimization algorithm were initiated using various start simplices positioned at different locations in the multi-dimensional parameter space. A variety of convergent solutions with comparably small standard deviations of the normal points relative to the fitted curves of order 0.005 mag were achieved. Since the system is not eclipsing, the light variations are almost entirely due to reflection and ellipsoidal variability. Therefore the reflectivity of the cool secondary atmosphere as measured by its bolometric albedo $A_{2}$ as well as the relative sizes of primary and secondary components are of crucial importance for the photometric solutions.

We actually found two groups of equally good solutions, which can be characterized by average values of $A_{2}$ close to $0.5( \pm 0.15)$ and $1.0( \pm 0.10)$, while the corresponding ratio of radii $r_{2} / r_{1}$ is $3.0( \pm 0.15)$ for the first group with smaller $A_{2}$, and $2.7( \pm 0.19)$ for the group with $A_{2} \approx 1.0$. The required amount of reflected light can hence be either provided by a larger secondary with relatively low reflectivity, or by a smaller star, which is reflecting light more efficiently. Therefore $A_{2}$ and those parameters, which determine the relative sizes of both components (mainly $\Omega_{1,2}$ ) are anti-correlated. From a numerical point of view, there is no way to decide which group of solutions is superior, because the theoretical curves of both groups are fitting the observations nearly indistinguishably well.

In principle, parameter correlations (e.g., between $i, q$ and $\Omega$ ) are especially severe in the case of a non-eclipsing system, and a multitude of comparably good solutions can be expected. Nevertheless, in this case the availability of spectroscopic information alleviates the confinement of physically reasonable parameter ranges. Our choice of solutions, which are considered as possible realistic representations of the binary, was made with due regard to boundary conditions deduced from our spectroscopic material and evolutionary scenarios discussed in detail in Sects. 4.4 and 5.

One important criterion was the comparison of photometrically suggested configurations with mass-radius relations for sdB and cool main sequence stars. Based on such consistency checks we sorted out unrealistic solutions and ended up with the two solution sets. As a quantitative measure of the fit quality we used the $1 \sigma$ deviation of all $B V R$ observations from the theoretical light curves. These $1 \sigma$ values of solutions within the two selected subsets all lie between 0.0054 and 0.0058 intensity units (i.e. between 0.0059 and $0.0063 \mathrm{mag}$ ).

Due to parameter correlations, the uncertainties of adjusted parameters in individual solutions are actually larger than implied by the formal fit errors . This is why we give instead standard deviations of a certain parameter from its mean value calculated from the 7 and 8 best solutions within the two solution groups characterized by $A_{2} \sim 0.5$ and $\sim 1.0$, respectively. For instance we find errors of order $\pm 6 \%$ for the orbital inclination $i$ and the photometric mass ratio $q$. The uncertainties in these quantities would otherwise be considerably larger, if boundary conditions limiting the physically reasonable parameter ranges would not have been considered.

In Table 2 we list representative examples for each of the two groups with different secondary albedo. The theoretical curves together with the individual observations are shown in Fig. 2. The synthetic $B V R$ curves are plotted for only one solution, because the deviations between both solution curves could hardly be graphically resolved, being about one order of magnitude less than the scatter of observational normal points. The $V$ and $R$ curves are offset relative to the $B$ curve by 0.1 and 0.2 intensity units, respectively. The light variability is mostly caused by a strong reflection effect with amplitudes of $0.21(B)$, $0.28(V)$, and $0.33(R)$. The overall run of the $B V R$ curves is reproduced without noticeable systematic deviations. The $\sigma$ value is smallest for the JKT $B$ photometry $(\sigma=0.003)$, and somewhat larger for the Hoher List $V(\sigma=0$. 006$)$ and $R$ $(\sigma=0$ m 008$)$ observations. In all cases, maximum deviations of normal points are below the $3 \sigma$ limit.

The best fit requires only a negligible amount of third light contribution in the $B$ and $R$ passbands of less than $0.5 \%$ (at quadrature). The $V$ curve is best represented for $l_{3} \sim 4 \%$. Such wavelength dependence of $l_{3}$ was also observed for another similar sdB binary with pronounced reflection effect HS 0705+6700 (Drechsel et al. 2001), in which a hot sdB primary $\left(T_{\text {eff }}=29000 \mathrm{~K}\right)$ causes strong heating of the facing side of a cool M dwarf $\left(T_{\text {eff }}=2900 \mathrm{~K}\right)$. Though bolometric reradiation of the incident flux can be expected, the complex absorption, reemission and scattering processes in the irradiated surface area might be responsible for a frequency redistribution, which can lead to some excess flux emerging in certain passbands (e.g., in the $V$ region).

The system with an orbital period of $4.12 \mathrm{~h}$ is found to be in a close detached configuration (actually nearly semi-detached in the case $A_{2}=0.39$ ). For this solution the radius of the secondary is 3.1 times larger than that of the sdB primary. Tidal and rotational deformation is weak for the primary, but the 
Table 2. Light curve solutions of HS 2333+3927.

\begin{tabular}{|c|c|c|}
\hline & $\begin{array}{c}A_{2}=0.39 \\
\sigma=0.00547\end{array}$ & $\begin{array}{c}A_{2}=1.00 \\
\sigma=0.00552\end{array}$ \\
\hline \multicolumn{3}{|c|}{ Fixed parameters: } \\
\hline$T_{1}$ & $36000 \mathrm{~K}$ & $36000 \mathrm{~K}$ \\
\hline$A_{1}{ }^{a}$ & 1.0 & 1.0 \\
\hline$g_{1}^{b}$ & 1.0 & 1.0 \\
\hline$g_{2}{ }^{b}$ & 0.32 & 0.32 \\
\hline$x_{1}(\mathrm{~B})^{c}$ & 0.27 & 0.27 \\
\hline$x_{1}(\mathrm{~V})^{c}$ & 0.25 & 0.25 \\
\hline$x_{1}(\mathrm{R})^{d}$ & 0.16 & 0.16 \\
\hline$x_{2}(\mathrm{~B})$ & 0.50 & \\
\hline$x_{2}(\mathrm{~V})$ & 0.50 & \\
\hline$x_{2}(\mathrm{R})$ & 0.50 & \\
\hline$\delta_{2}^{e}$ & 0.0 & 0.0 \\
\hline \multicolumn{3}{|c|}{ Adjusted parameters: } \\
\hline$i$ & $39.6 \pm 1.9$ & $36.6 \pm 2.2$ \\
\hline$q\left(=\mathrm{M}_{2} / \mathrm{M}_{1}\right)$ & $0.736 \pm 0.043$ & $0.744 \pm 0.046$ \\
\hline$\Omega_{1}$ & $9.5 \pm 0.6$ & $12.0 \pm 0.4$ \\
\hline$\Omega_{2}$ & $3.4 \pm 0.3$ & $4.5 \pm 0.3$ \\
\hline$A_{2}$ & $0.39 \pm 0.14$ & $1.00 \pm 0.10$ \\
\hline$T_{\text {eff }}(2)$ & $3800 \mathrm{~K} \pm 800 \mathrm{~K}$ & $3400 \mathrm{~K} \pm 600 \mathrm{~K}$ \\
\hline$L_{1}(B)^{f}$ & $0.998 \pm 0.001$ & $0.999 \pm 0.001$ \\
\hline$l_{3}(B)^{g}$ & $0.2 \% \pm 0.2 \%$ & $0.5 \% \pm 1.0 \%$ \\
\hline$L_{1}(V)^{f}$ & $0.991 \pm 0.001$ & $0.996 \pm 0.003$ \\
\hline$l_{3}(V)^{g}$ & $4.4 \% \pm 1.4 \%$ & $3.6 \% \pm 1.6 \%$ \\
\hline$L_{1}(R)^{f}$ & $0.982 \pm 0.003$ & $0.992 \pm 0.007$ \\
\hline$l_{3}(R)^{g}$ & $0.4 \% \pm 0.7 \%$ & $0.0 \% \pm 0.3 \%$ \\
\hline$x_{2}(\mathrm{~B})$ & & $0.54 \pm 0.15$ \\
\hline$x_{2}(\mathrm{~V})$ & & $0.48 \pm 0.07$ \\
\hline$x_{2}(\mathrm{R})$ & & $0.50 \pm 0.11$ \\
\hline$\delta_{1}^{e}$ & $0.024 \pm 0.004$ & $0.029 \pm 0.006$ \\
\hline \multicolumn{3}{|l|}{ Roche radii: ${ }^{h}$} \\
\hline$r_{1}$ (pole) & $0.112 \pm 0.012$ & $0.086 \pm 0.004$ \\
\hline$r_{1}$ (point) & $0.112 \pm 0.012$ & $0.086 \pm 0.004$ \\
\hline$r_{1}($ side $)$ & $0.112 \pm 0.012$ & $0.086 \pm 0.004$ \\
\hline$r_{1}($ back $)$ & $0.112 \pm 0.012$ & $0.086 \pm 0.004$ \\
\hline$r_{2}($ pole $)$ & $0.318 \pm 0.029$ & $0.222 \pm 0.010$ \\
\hline$r_{2}$ (point) & $0.376 \pm 0.051$ & $0.228 \pm 0.012$ \\
\hline$r_{2}($ side $)$ & $0.332 \pm 0.032$ & $0.225 \pm 0.011$ \\
\hline$r_{2}$ (back) & $0.357 \pm 0.039$ & $0.229 \pm 0.011$ \\
\hline
\end{tabular}

${ }^{a}$ Bolometric albedo.

${ }^{b}$ Gravitational darkening exponent.

${ }^{c}$ Linear limb darkening coefficient; theoretical value taken from Díaz-Cordovés (1995).

${ }^{d}$ Linear limb darkening coefficient; theoretical value taken from Wade \& Rucinski (1985).

${ }^{e}$ Radiation pressure parameter, see Drechsel et al. (1995).

${ }^{f}$ Relative luminosity $L_{1} /\left(L_{1}+L_{2}\right)$.

${ }^{g}$ Fraction of third light at maximum.

${ }^{h}$ Fractional Roche radii in units of separation of mass centers. secondary is appreciably distorted: the ratio of radii in direction of the inner Lagrangean point relative to that in polar direction, $r_{\text {point }} / r_{\text {pole }}$, is close to unity for the primary, namely 1.003 , but amounts to 1.182 for the secondary. 3D-simulated aspects of the system at orbital phases $0.0,0.25$, and 0.5 as viewed under its inclination angle of $39^{\circ} .6$ and $36^{\circ} 6$, respectively, for both solutions are shown in Fig. 3. Further information from spectroscopy is needed to better constrain the system parameters.

\section{Spectroscopic analysis}

The optical spectra allow us to measure the radial velocity curve with good phase coverage as well as a quantitative spectral analysis to determine the atmospheric parameters and the projected rotational velocity.

\subsection{Radial velocity curve}

Using the MIDAS long slit context, radial velocities were determined from the TWIN spectra from a fit of a set of mathematical functions to the observed line profiles. In most blue spectra we fitted the profiles of the He I line at $4471.60 \AA$ and the He II line at $4685.70 \AA$. Within windows of $50 \AA$ width centered on the rest wavelengths single Gaussians were fitted to the lines, and a linear function to reproduce the overall spectral trend. For three stars the two ThAr exposures bracketing their exposures in the blue channel of the twin spectrograph yielded inconsistent wavelength solutions, suggesting an instrumental problem. In these cases $\mathrm{H} \alpha$ in the red part of the spectrum was used for the radial velocity determination instead of the He lines. The $\mathrm{H} \alpha$ line core was fitted with a Gaussian, and again a linear function within a $100 \AA$ wide region around the line center was used to reproduce the continuum. Since the $\mathrm{H} \alpha$ line is very broad, an additional Lorentzian was used to model the line wings. The radial velocity itself was calculated from the central wavelength of the fitted Gaussian for both $\mathrm{H} \alpha$ and He-lines. The measured radial velocities were then corrected to heliocentric values.

To perform an independent determination of the orbital period from the spectroscopic data and to verify the photometric ephemeris, the measured radial velocities (after heliocentric correction of velocity values and mid-exposure times) were subjected to a periodogram analysis. Minimum $\chi^{2}$ was found very close to the photometric period at $P=14844.12 \mathrm{~s}$. The peak width in the power spectrum is, of course, much broader than for the photometric determination because of the shorter duration of the spectroscopic run and the intrinsic errors of the velocity measurements.

Since this system is single-lined and orbits are circular, the analysis of the radial velocity curve is straightforward. A comparison of the best fit sine curve and the measured radial velocities is shown in Fig. 4.

From the radial velocity curve (Fig. 4) we found the semiamplitude to be

$K_{1}=89.6 \pm 3.2 \mathrm{~km} \mathrm{~s}^{-1}$

and a systemic velocity of $\gamma=-31.4 \pm 2.1 \mathrm{~km} \mathrm{~s}^{-1}$. 


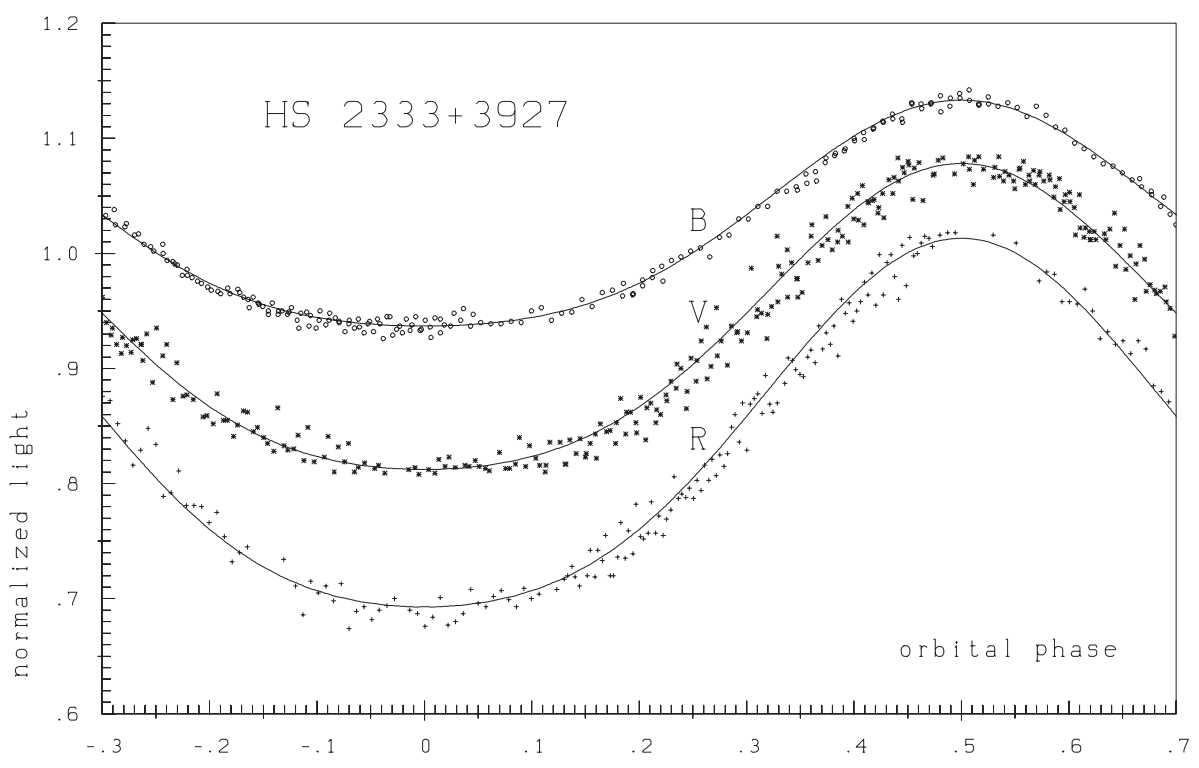

Fig. 2. $B V R$ light curves of HS $2333+3927$ obtained on July 25-29, $2002(B)$ at JKT, La Palma, Oct. 8-10 (V) and Aug. 17-18, 2002 (R) at Hoher List, Bonn; the solid lines represent the best fit solution (for $A_{2}=0.39$ ) according to the parameters listed in Table 2; $V$ and $R$ curves are offset relative to $B$ curve by 0.1 and 0.2 intensity units.

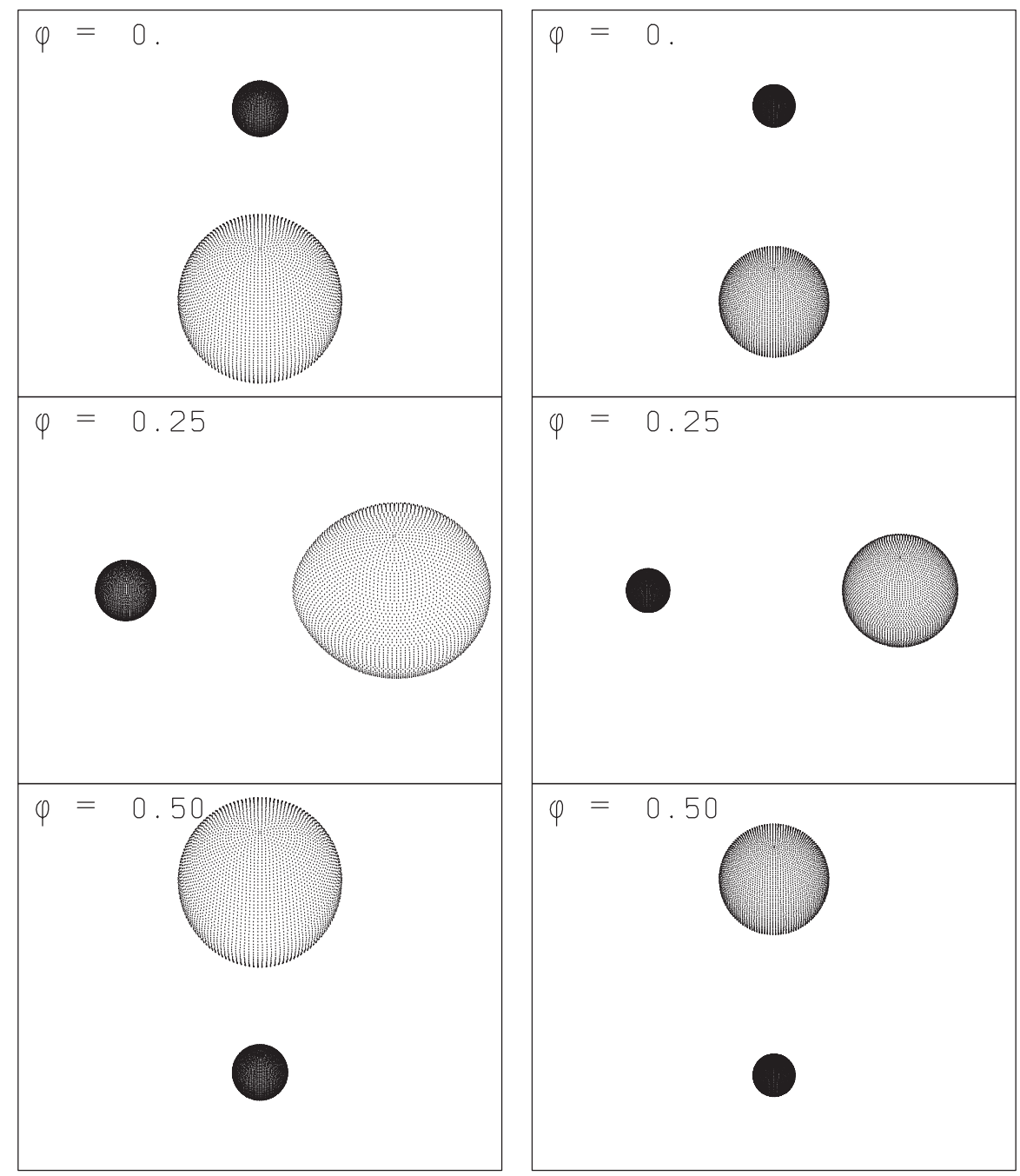

Fig. 3. Aspects of HS $2333+3927$ at orbital phases 0.0, 0.25, and 0.50, viewed under the orbital inclination angle of $39^{\circ} .6$ for the low albedo $\left(A_{2}=0.39\right)$ solution (left part) and $36^{\circ} .6$ for the $A_{2}=1.0$ solution (right part); the larger star is the cool M dwarf secondary. 


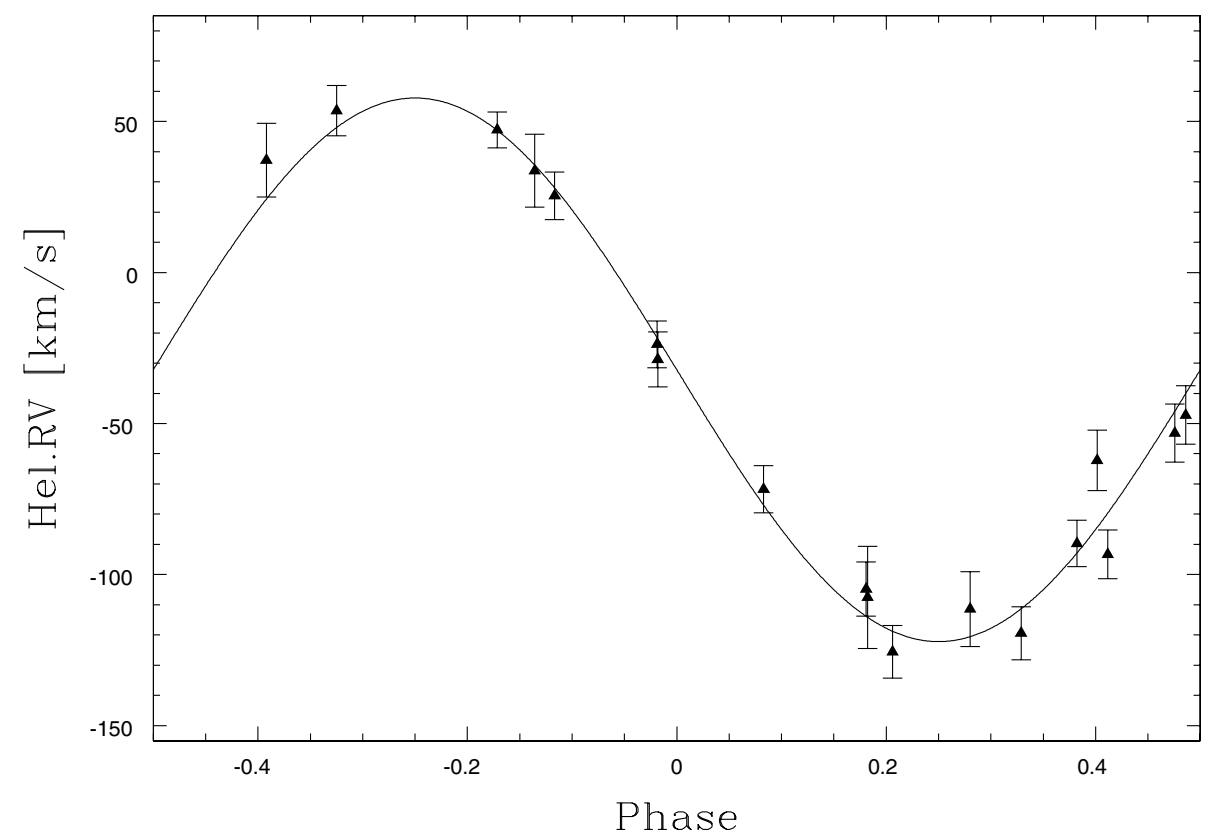

Fig. 4. Radial velocity curve of HS 2333+3927. Phases were calculated using the ephemeris derived in Sect. 3.1. The semi-amplitude amounts to $K_{1}=89.6 \pm 3.2 \mathrm{~km} \mathrm{~s}^{-1}$, and the systemic velocity is $\gamma=-31.4 \pm 2.1 \mathrm{~km} \mathrm{~s}^{-1}$; error bars correspond to $3 \sigma$ margins.

The mass function can be computed from the semi-amplitude and the period to be

$f(m)=\frac{A^{3} P}{2 \pi G}=0.0128 M_{\odot} \pm 0.0013$.

\subsection{Line profile variations}

Close inspection of the red spectra revealed that the $\mathrm{H} \alpha$ line profile varies with phase (see Fig. 5). We also plot the photospheric $\mathrm{H} \alpha$ line profile computed from the model atmosphere that fits the blue spectrum best (see Sect. 4.3). As can be seen the synthetic profile matches the observation reasonably well near lower conjunction $(\phi \approx 0.0)$. Near upper conjunction $(\phi \approx$ $0.5)$ the line core is shallower than predicted, which we attribute to emission from the heated hemisphere of the companion filling in the absorption line of the sdB star. Some redshifted emission may also be discernible at phases between quadrature and upper conjunction $(\phi=0.276 \ldots 0.481)$ in Fig. 5 .

\subsection{Spectrum fitting}

The Balmer and helium lines in blue spectra can be used to determine the atmospheric parameters by performing a quantitative spectral analysis. Line profile variations such as observed for $\mathrm{H} \alpha$ do not become evident for the higher Balmer lines or for the helium lines by visual inspection of the blue spectra.

Matching the synthetic Balmer $(\mathrm{H} \beta$ to $\mathrm{H} \epsilon)$ and $\mathrm{He} \mathrm{I}$ (4471 $\AA$ and $4922 \AA$ ) as well as He II $4686 \AA$ line profiles to the observations resulted in a determination of effective temperature, gravity and He abundance. It turned out that the observed line cores are broader than predicted. Therefore, we had to consider rotational broadening as well. A projected rotational velocity of $v_{\text {rot }} \sin i=75 \mathrm{~km} \mathrm{~s}^{-1}$ was adopted, which, however, is not well constrained by the spectral fits.
Table 3. Heliocentric radial velocities of HS 2333+3927. Heliocentrically corrected times (HJD) are given for mid-exposures. Phases $\Phi$ are computed from the ephemeris given in Sect. 3.1. The fitted lines are listed in the last column.

\begin{tabular}{ccrl}
\hline $\begin{array}{c}\text { HJD (mid-exp.) } \\
-2452000\end{array}$ & $\Phi$ & $\begin{array}{r}\text { hel. RV } \\
\left(\mathrm{km} \mathrm{s}^{-1}\right)\end{array}$ & lines \\
\hline 498.6097 & 0.414 & $-93 \pm 8$ & He I, He II \\
498.6874 & 0.866 & $34 \pm 12$ & He I, He II \\
499.4291 & 0.183 & $-105 \pm 9$ & He I, He II \\
499.4815 & 0.488 & $-47 \pm 10$ & He I, He II \\
499.5403 & 0.831 & $47 \pm 6$ & H $\alpha$ \\
499.6052 & 0.208 & $-126 \pm 9$ & H $\alpha$ \\
499.6388 & 0.404 & $-62 \pm 10$ & H $\alpha$ \\
499.6743 & 0.610 & $37 \pm 12$ & He I, He II \\
501.4566 & 0.984 & $-29 \pm 9$ & He I, He II \\
501.5757 & 0.678 & $54 \pm 8$ & He I, He II \\
502.5470 & 0.333 & $-119 \pm 9$ & He I, He II \\
505.5630 & 0.887 & $25 \pm 8$ & He I, He II \\
505.5798 & 0.984 & $-24 \pm 8$ & He I, He II \\
505.5973 & 0.086 & $-72 \pm 8$ & He I, He II \\
505.6144 & 0.185 & $-108 \pm 17$ & He I, He II \\
505.6312 & 0.283 & $-111 \pm 12$ & He I, He II \\
505.6487 & 0.385 & $-90 \pm 8$ & He I, He II \\
505.6648 & 0.479 & $-53 \pm 10$ & He I, He II \\
\hline
\end{tabular}

Because the medium resolution TWIN spectra cover only few of the Balmer lines, we used the CAFOS spectra which cover the Balmer series to its limit. Since the high Balmer lines are probably least affected by reprocessed light from the secondary, much emphasis was put in the fit procedure to 


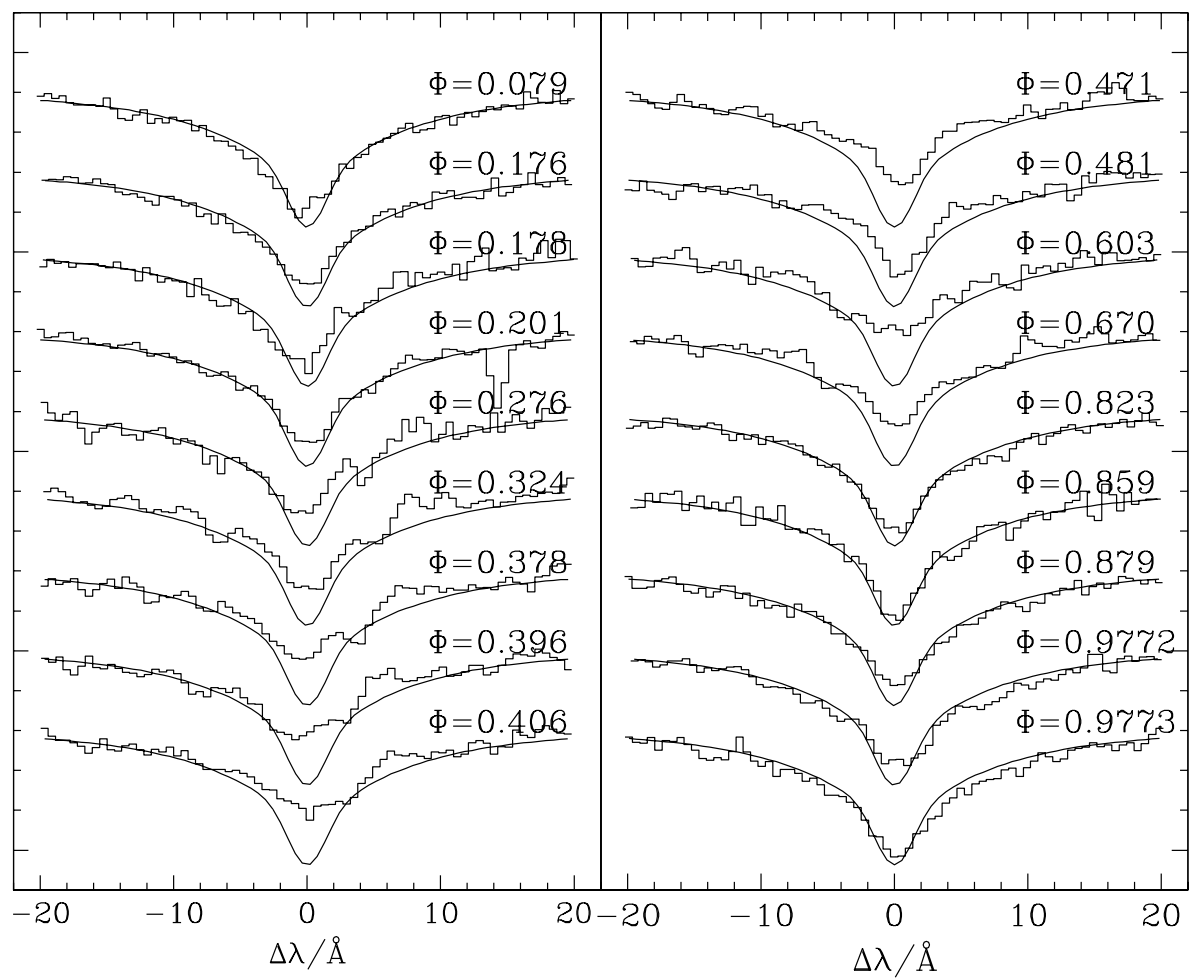

Fig. 5. Variation of the $\mathrm{H} \alpha$ line profile with phase. The predicted line profile calculated from a model with $T_{\text {eff }}=36500 \mathrm{~K}$, $\log g=5.7$ and $\log (\mathrm{He} / \mathrm{H})=-2.15$ is overlayed to each observation.

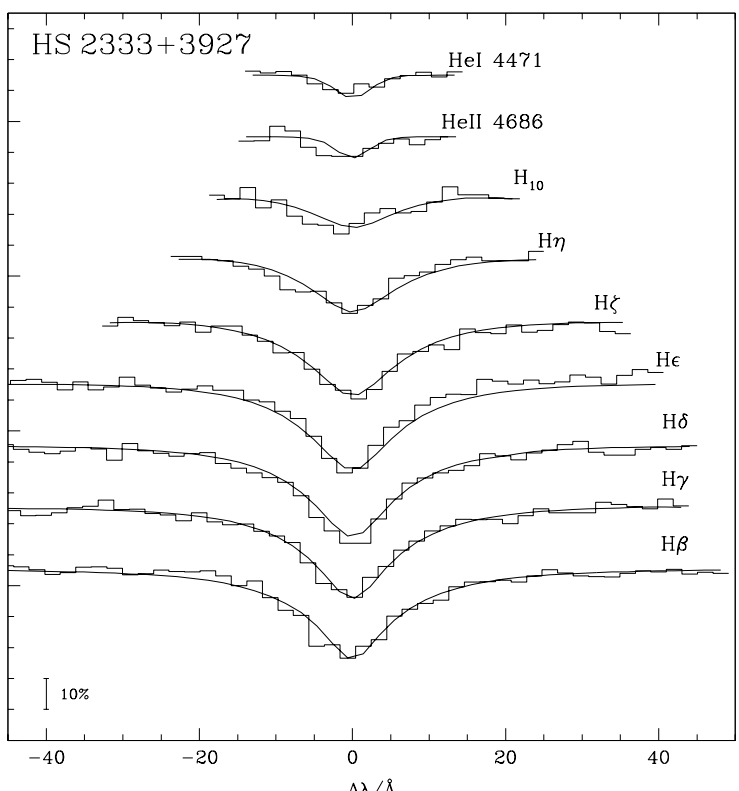

Fig. 6. Fit of the Balmer and helium lines in a typical CAFOS spectrum of HS $2333+3927$ by synthetic spectra calculated from NLTE model atmospheres. A projected rotational velocity of $75 \mathrm{~km} \mathrm{~s}^{-1}$ is adopted.

reproduce these lines well. The results for the 13 available phases are summarized in Table 4, and are plotted in Fig. 7. The errors listed in Table 4 and plotted in Fig. 7 are statistical ones and are unrealistically low. A strong variation of $T_{\text {eff }}$ with phase by up to $6000 \mathrm{~K}$ becomes apparent (upper panel of Fig. 7), the highest temperatures occurring near upper
Table 4. Atmospheric parameters of HS 2333+3927.

\begin{tabular}{llcc}
\hline \hline phase & $T_{\text {eff }}(\mathrm{K})$ & $\log g$ & $\log (\mathrm{He} / \mathrm{H})$ \\
\hline 0.015 & $36236 \pm 21$ & $5.65 \pm 0.01$ & $-2.01 \pm 0.15$ \\
0.081 & $37204 \pm 200$ & $5.71 \pm 0.02$ & $-2.26 \pm 0.26$ \\
0.147 & $36947 \pm 169$ & $5.70 \pm 0.01$ & $-2.35 \pm 0.21$ \\
0.213 & $38663 \pm 100$ & $5.63 \pm 0.01$ & $-2.34 \pm 0.20$ \\
0.279 & $39074 \pm 173$ & $5.60 \pm 0.03$ & $-2.71 \pm 0.27$ \\
0.344 & $41840 \pm 211$ & $5.74 \pm 0.02$ & $-2.26 \pm 0.23$ \\
0.410 & $41600 \pm 294$ & $5.66 \pm 0.01$ & $-2.53 \pm 0.26$ \\
0.479 & $39694 \pm 241$ & $5.78 \pm 0.01$ & $-2.46 \pm 0.24$ \\
0.545 & $39161 \pm 356$ & $5.74 \pm 0.03$ & $-2.24 \pm 0.15$ \\
0.610 & $40620 \pm 441$ & $5.81 \pm 0.04$ & $-2.27 \pm 0.15$ \\
0.677 & $38932 \pm 80$ & $5.54 \pm 0.03$ & $-2.27 \pm 0.14$ \\
0.883 & $36450 \pm 42$ & $5.67 \pm 0.01$ & $-2.37 \pm 0.19$ \\
0.949 & $36482 \pm 46$ & $5.64 \pm 0.01$ & $-2.35 \pm 0.18$ \\
\hline
\end{tabular}

conjunction. The gravity varies little, only by about $\pm 0.1 \mathrm{dex}$ (lower panel of Fig. 7), which we adopted as a realistic estimate of its error.

Since the spectrum displays helium lines from two stages of ionization, we can use the ionization equilibrium of helium to determine $T_{\text {eff }}$. Inspection of the spectral line fits for phases near upper conjunction $(\phi=0.5)$ reveals that the helium lines are very poorly matched. Therefore we removed the Balmer lines from the fitting process and determined $T_{\text {eff }}$ and 

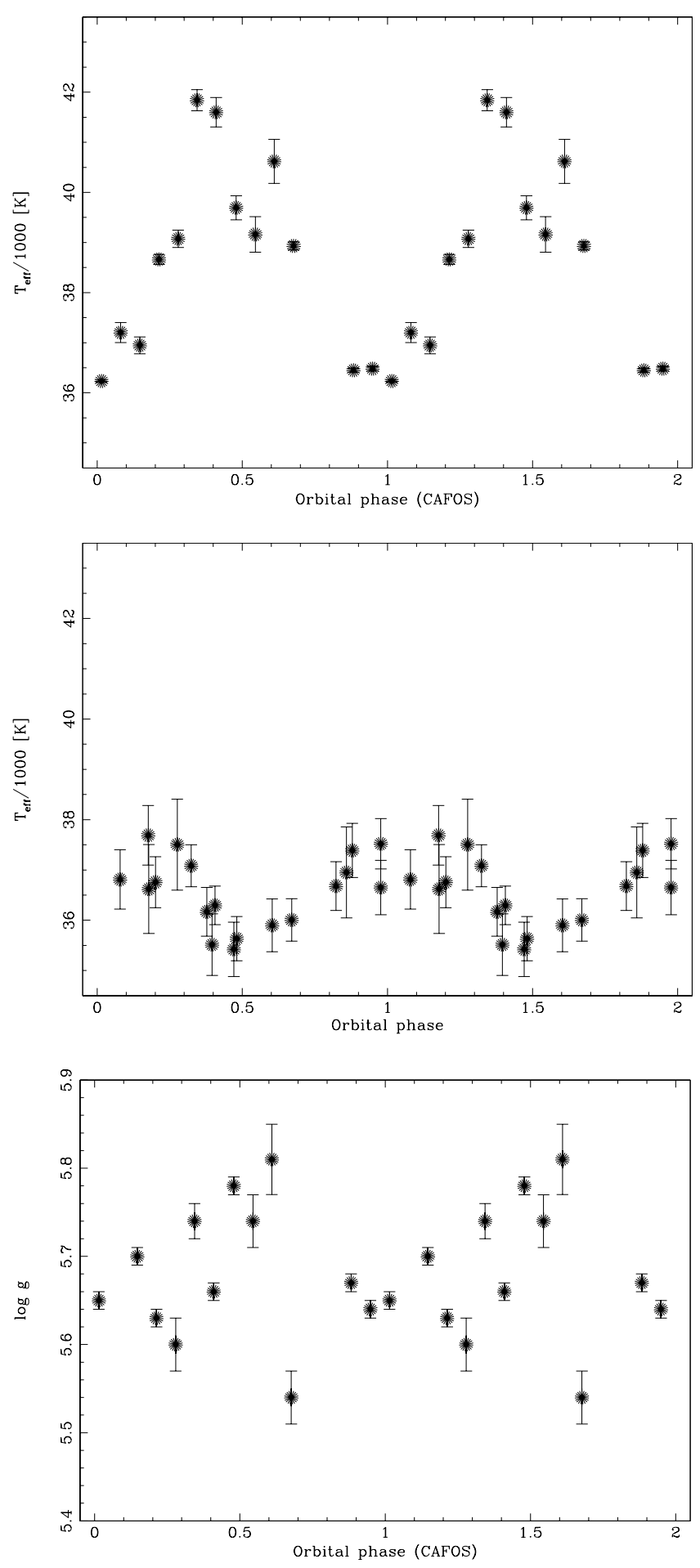

Fig. 7. Variation of atmospheric parameters with phase; the error bars result from the $\chi^{2}$ fit procedure described by Napiwotzki et al. (1999).

helium abundance from the helium lines only. Since the helium lines are insensitive to gravity, the gravity was fixed to $\log g=$ 5.7 .

The results are shown in the middle panel of Fig. 7. The variation of $T_{\text {eff }}$ is also present, but at a much smaller amplitude (about $2000 \mathrm{~K}$ ) than found from the Balmer lines. As can be seen by comparing the upper and the middle panel of Fig. 7 the temperature variations are countercurrent to that derived from the Balmer lines, i.e. the highest $T_{\text {eff }}$ occurs near lower conjunction and the lowest near upper conjunction.

From Fig. 7 we conclude that the temperature variations are not real, but caused by reprocessed light from the secondary contaminating the photospheric spectrum of the sdB star. Apparently, the helium lines are less affected than the Balmer lines. The atmospheric parameters determined from spectra taken near lower conjunction should be least affected by reprocessed light from the secondary and, therefore, the atmospheric parameters derived from them should be the closest approximation to the true atmospheric parameters of the sdB star. The helium ionization equilibrium indicates $T_{\mathrm{eff}} \approx 37000 \mathrm{~K}$ near $\phi=0.0$, whereas the Balmer lines indicate a slightly lower $T_{\text {eff }}$ of $36000 \mathrm{~K}$. As a compromise we used $T_{\text {eff }}=36500 \pm$ $1000 \mathrm{~K}$.

Based on the above consideration we finally adopted $T_{\text {eff }}=36500 \pm 1000 \mathrm{~K}, \log g=5.70 \pm 0.1$ and $\log \left(n_{\mathrm{He}} / n_{\mathrm{H}}\right)=-2.15 \pm 0.15$ for the atmospheric parameters of HS $2333+3927$.

\subsection{Mass-radius relations}

The light curve analysis provided us with an estimate of the system's inclination. For any assumed primary mass we can calculate the secondary mass from the mass function. In addition the radius of the orbit $a_{1}$ of the primary can be calculated using $a_{1}=13751 \times K_{1} \times P / \sin i$ (assuming a circular orbit). The separation A of the components can then be calculated from the mass ratio $\mathrm{q}$ by $A=(1+1 / q) \times a_{1}$. The light curve allowed us to determine the ratio of the radii in units of the separation. Hence we can determine the radii of both components for any given sdB mass, i.e. we can derive mass-radius relations for both components. These can be compared to independently determined relations. For the sdB star such a relation follows from the surface gravity. For the cool companion the mass-radius relation for low mass main sequence stars may be appropriate. The mass of the sdB star could then be determined by requesting these mass-radius relations to be consistent.

Figure 8 compares the mass-radius relation of the sdB derived from the light and radial velocity curves for two values of the albedo of the cool companion $\left(A_{2}\right)$ to that derived from gravity. The gravity of $\log g=5.7 \pm 0.1$ is derived in Sect. 4.3. For the solution for a high secondary albedo the M-R-relation from light and radial velocity curve intersects the one from gravity for unreasonably low masses of 0.08 to 0.15 . In the case of a lower secondary albedo the range of allowed sdB masses is 0.15 to $0.35 M_{\odot}$. This is still considerably lower than the canonical sdB mass of $0.47 M_{\odot}$. Such a mass would require the gravity to be higher by $0.16 \operatorname{dex}\left(A_{2}=0.39\right)$ or even $0.38 \operatorname{dex}\left(A_{2}=1.0\right.$, see Fig. 8$)$. Due to the contamination of the sdB's Balmer line spectrum used to derive the gravity with reflected light from the secondary, it is possible that the sdB gravity is somewhat underestimated (see Sect. 4.3). A similar discrepancy has been noted for the eclipsing sdOB binary AA Dor (Rauch 2000; Hilditch et al. 2003).

Further constraints can be drawn from the mass-radius relation for the companion. However, for the secondary the 


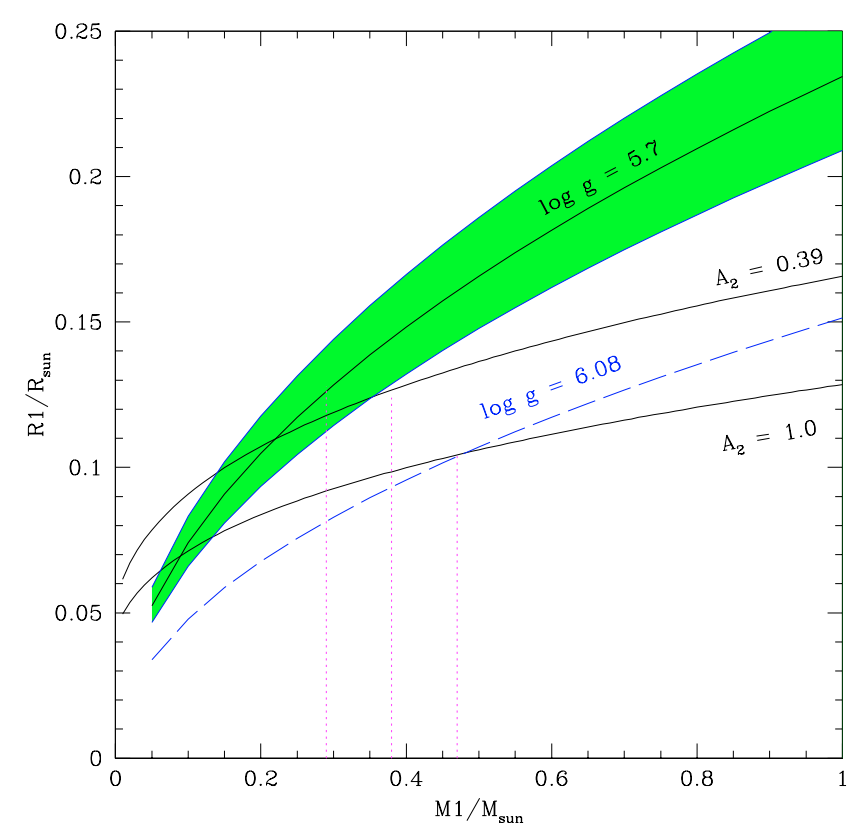

Fig. 8. Comparison of the sdB mass-radius relation from the analysis of light and radial velocity curve (full drawn line) to those derived from gravities: those from the spectroscopic fit $\log g=5.7 \pm$ 0.1 (shaded area and one for a hypothetical higher gravity of $\log g=$ 6.08. The positions of sdBs for masses of $0.29 M_{\odot}$ (as predicted by post-RGB evolutionary models), of $0.38 M_{\odot}$ (the lowest sdB mass for core helium burning, Han et al. 2003) and of $0.47 M_{\odot}$ (the canonical mass) are marked by the vertical lines.

gravity is unknown. Therefore we have to use a mass-radius relation from observations of normal $\mathrm{M}$ dwarfs or predictions from evolutionary models. We used model predictions by Baraffe \& Chabrier (1996) and the observational relation measured by Clemens et al. (1998, see Fig. 9). For a high albedo $A_{2}=1.0$ the M-R-relation for the companion of HS 2333+3927 lies below these relations, while it lies above it for $A_{2}=0.39$. Since the secondary star is strongly heated by the sdB star and may even be distorted (see Fig. 3) it probably does not obey the mass-radius relation for normal M stars. It seems plausible that the star is larger than a normal $\mathrm{M}$ star at a given mass, which would argue against the high albedo solution.

In summary, the mass-radius relations discussed above favour strongly the low albedo solution. Accordingly the mass of the sdB star is small, i.e. 0.15 to $0.35 M_{\odot}$, but the canonical sdB mass of $0.47 M_{\odot}$ cannot be excluded yet, because the gravity would need to be higher by 0.16 dex, only (see Fig. 8).

\section{Evolution}

In Fig. 10 we compare the position of HS2333+3927 as well as four similar systems with a sample of close binary sdB stars recently studied by Morales-Rueda et al. (2003) and with calculations for EHB evolution from Dorman et al. (1993). HS2333+3927 lies at the hot edge of the distribution and is consistently explained by a post-EHB track of $0.47 M_{\odot}$. There are two viable scenarios to explain the formation of HS2333+3927: the Han et al. (2003) and the post-RGB

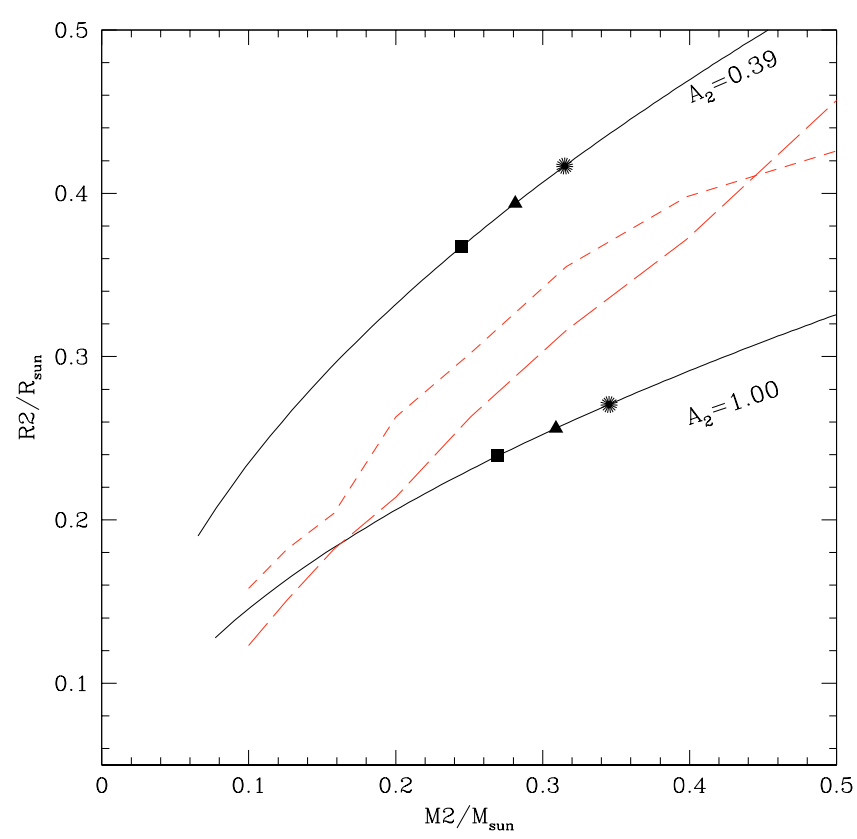

Fig. 9. Comparison of the cool companion's mass-radius relations derived from the analysis of light and radial-velocity curves for two albedos of the secondary $\left(A_{2}=0.39\right.$ and $A_{2}=1.0$, respectively, see text $)$ to that of observed M-type main sequence stars (short-dashed; Clemens et al. 1998) and to a theortical one (dashed; Baraffe \& Chabrier 1996). The filled square marks the position for which the sdB star has a mass of $0.29 M_{\odot}$ (as predicted by post-RGB evolutionary models), the filled circle marks the position for which the sdB primary has the canonical mass of $0.47 M_{\odot}$ (as predicted by EHB evolutionary models) and the filled triangle the lowest $\mathrm{sdB}$ mass for core helium burning (Han et al. 2003).

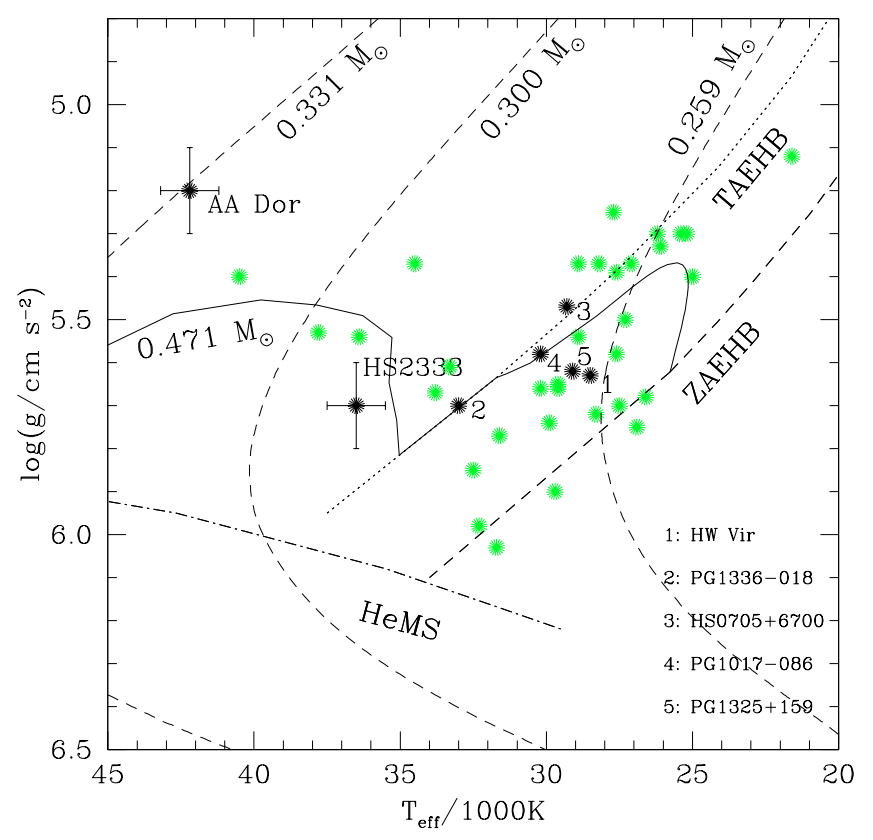

Fig. 10. Comparison of the position of HS $2333+3927$ in the $\left(T_{\text {eff }}\right.$, $\log g$ ) diagram to that of HW Vir, PG 1336-018, HS 0705+6700, PG 1017-086, PG 1325+159 and AA Dor and other sdB binaries with known periods as listed in Morales-Rueda et al. (2003). An evolutionary track for post-EHB evolution by Dorman et al. (1993) labeled with the stellar mass is also shown as well as three tracks for post-RGB evolution (Driebe et al. 1998) also labeled by their stellar masses. 
scenario, which will be described in detail below (see also Heber 2003).

\subsection{The Han et al. scenario}

The channels by which core helium burning sdB stars can form within a binary system have been studied by Han et al. (2002, 2003):

(i) The "common envelope (CE) ejection (CEE) channel" can be divided into two others. In the "first CEE channel", the initially more massive star experiences mass transfer when it is a red giant. This results in the formation of a CE, the spiraling-in of both stars and consequently the ejection of the $\mathrm{CE}$ leading to the formation of a very close binary. If the core of the giant star still ignites helium it becomes an sdB. The final result is therefore an $\mathrm{sdB}$ star in a short period binary with a main sequence companion. The "second CEE channel" is very similar to the first CE ejection channel, but in this case the initially more massive star is already a white dwarf. This channel can lead to shorter orbital periods, because a white dwarf has a smaller radius than a main-sequence star and can penetrate deeper into the $\mathrm{CE}$ without merging. SdB stars that form through this second channel have a wider range of orbital periods and their companions are white dwarfs.

(ii) The second channel is the "stable Roche lobe overflow (RLOF) channel" and involves stable mass transfer, where a low-mass giant fills its Roche lobe on the red giant branch and loses most of its envelope as a result of stable RLOF. As in the CEE case, a RLOF case could occur a second time when the initially more massive star is already a white dwarf, but is found to be negligible for the sdB population.

(iii) The third channel is the "merger channel", i.e. sdB stars can be formed by the merger of two helium white dwarfs, if the mass is sufficient to burn helium in the core. Consequently the resulting sdB should be single.

The investigation of Han et al. (2003) also predicts a mass distribution for $\mathrm{sdB}$ stars peaked at the canonical sdB mass of $0.46 M_{\odot}$ for single star evolution (Dorman et al. 1993), but much wider than assumed previously with masses ranging from $\approx 0.38 M_{\odot}$ to $\approx 0.8 M_{\odot}$, if selection bias is taken into account. The highest masses are mostly due to mergers, while the lowest masses result from first stable Roche lobe overflow. Since the companion of HS $2333+3927$ is a low mass main-sequence star the system has been formed during the first mass transfer phase. The progenitor of the sdB star must have had a main sequence mass of at least $0.8 M_{\odot}$ in order to have evolved off the main sequence in less than a Hubble time. Hence the primordial mass ratio of the system is larger than $q=2$, which rules out stable Roche lobe overflow (Han et al. 2003). Therefore the system must have been formed through the first CEE phase. The mass range allowed by the mass-radius relation for the $\mathrm{sdB}$ primary would not rule out this scenario, although the lowest mass for core helium burning stars in the Han et al. models is at the upper limit of the former.

\subsection{The post-RGB scenario}

There is another possibility to form hot subluminous stars of low mass in binaries. When the CEE ejection on the first giant branch occurs at helium core masses too low to ignite helium core burning, the primary will evolve as helium star through the sdB domain to form a helium core white dwarf (Driebe et al. 1998; Heber 2003). We will refer to this kind of evolution as the post-RGB scenario. It has been successfully invoked to explain the low mass of $0.2 M_{\odot}$ of the sdB primary of HD 188112 (Heber et al. 2003b). Rauch (2000) argues that this post-RGB evolution also holds for the eclipsing sdOB binary AA Dor, and derives a mass of $0.33 M_{\odot}$. We compare the position of HS2333+3927 in the $\left(T_{\text {eff }}, \log g\right)$ plane (Fig. 10) to the evolutionary tracks of Driebe et al. (1998) for masses of $0.259 M_{\odot}$, $0.30 M_{\odot}$ and $0.331 M_{\odot}$. By interpolation we estimate a mass of $0.29 M_{\odot}$ for HS2333+3927. Such a low mass is in reasonable agreement with the sdB mass-radius relations for a low albedo of the secondary (see Fig. 8).

\subsection{Discussion}

Both evolutionary scenarios (the Han et al. and the post-RGB scenario) can explain the position of HS2333+3927 in the $\left(T_{\text {eff }}\right.$, $\log g$ ) diagram. The sdB mass predicted by the evolutionary models is much larger than the one derived from the massradius relation for a high albedo (see Fig. 8). In addition, the radius of the secondary is too small (see Fig. 9). Therefore the high albedo can be discarded. For the low albedo the sdB mass suggested by evolutionary theory agrees reasonably well with that from the mass-radius relations (see Fig. 8). The lower mass $\left(0.29 M_{\odot}\right)$ predicted by the post-RGB scenario is in better agreement with the mass-radius relation derived from the light and radial velocity curves. Since systematic errors may lead to an underestimate of the gravity, we cannot rule out an sdB mass as large as the canonical one. Therefore, we adopt a plausible mass range for the sdB between $0.29 M_{\odot}$ and $0.47 M_{\odot}$. Accordingly, the mass of the secondary is between $0.24 M_{\odot}$ and $0.32 M_{\odot}$, indicating a spectral type of M 3 to M 4 (Drilling \& Landolt 2000).

\section{Conclusions}

During a search for non-radial pulsations of sdB stars we discovered that HS $2333+3927$ is variable in light on a time scale much larger than expected for such oscillations. Extended light curve measurements showed that the variations are periodic $(P=0.1718023 \mathrm{~d} \pm 0.0000009)$. Radial velocity variations at the same period were detected, indicating that it is a binary system and the light variations are caused by the reflection effect. No eclipses are observed. The analysis of the light curve did not yield a unique solution, mainly because the albedo of the secondary is poorly constrained. Two solutions of equal quality with a high $\left(A_{2}=1.0\right)$ and a low $\left(A_{2}=0.39\right)$ albedo were considered further. The companion does not contribute to the optical light of the system except through a strong reflection effect. Variability of the Balmer line profiles, most notably for $\mathrm{H} \alpha$, was discovered. Because they correlate with 
phase, the profile distortions are probably caused by the reflection effect. A spectroscopic analysis of the presumably least affected blue spectra results in $T_{\text {eff }}=36500 \mathrm{~K}, \log g=5.70$, and $\log \left(n_{\mathrm{He}} / n_{\mathrm{H}}\right)=-2.15$. These characteristics are typical for $\mathrm{sdB}$ stars. Mass-radius relations are derived from the results of the analysis of light and radial velocity curves. Comparison with the observed mass-radius relation of the sdB star and with that of lower main sequence stars for the companion rules out the high albedo solution derived from the light curve analysis, because the mass of the sdB primary and the radius of the secondary are unrealistically low. The low albedo of $A_{2}=0.39$ we find for HS $2233+3927$ is in line with predictions for convective envelopes of cool stars. Rucinski (1969) first predicted that convection should reduce the flux from the illuminated hemisphere by about half the amount of the incident energy, i.e. an albedo of about 0.5. This assumes low illumination, for higher illumination the reduction would be even stronger (for a review see Vaz 1985). Note, however, that in the light curve analyses of other eclipsing sdB systems, e.g. HS $0705+6700$ (Drechsel et al. 2001) or AA Dor (Hilditch et al. 2003) the albedos were found to be very high (around 1.0). The secondary of HS 2333+3927 is more massive than the other ones (see below) and therefore its effective temperature is higher, which may explain why its albedo is lower than that of the others.

The low albedo solution and a discussion of plausible evolutionary scenarios suggest that the mass of the $\mathrm{sdB}$ lies between $0.29 M_{\odot}$ and $0.47 M_{\odot}$ and that of the secondary between $0.24 M_{\odot}$ and $0.32 M_{\odot}$. Accordingly the secondary is a main sequence star of spectral type M 3 to M 4. The mass of the secondary of HS $2333+3927$ is larger than in the four eclipsing systems which have $0.066 M_{\odot}$ (AA Dor, Rauch 2000), $0.134 M_{\odot}$ (HS 0705+6700), $0.17 M_{\odot}$ (PG 1336-018) and $0.18 M_{\odot}$ (HW Vir), respectively. HS $2333+3927$ is only the sixth $\mathrm{sdB}+\mathrm{dM}$ system discovered so far besides the three eclipsing systems and the non-eclipsing reflection effect binaries PG 1017-086 (Maxted et al. 2002) and PG 1329+159 (Maxted et al. 2003). Four closely related objects including AA Dor are discussed by Heber (2003, 2003b). Ongoing searches for reflection effects in short period sdB binaries will undoubtedly lead to new discoveries.

In order to further restrict the masses, an improved determination of the gravity is needed. A promising option is the measurement and analysis of the Lyman line profiles, because they should be unaffected by the reflected light from the companion. Since the system is not eclipsing the inclination angle is not easily determined. An estimate independent of the light curve analysis could be derived, if the projected rotational velocity could be measured reliably (as demonstrated by Drechsel et al. 2001 and Maxted et al. 2002). This was not possible from the optical spectra available. The analysis of metal lines in the UV and FUV, however, would allow an accurate measurement for the same reason as for the Lyman lines. Far-UV observations with HST and FUSE to measure these spectral lines are therefore urgently needed.

Finally, we consider the period distribution of known sdB binaries (Fig. 11, drawn from Morales-Rueda et al. 2003). Note that the companions are not always main sequence stars, but also white dwarfs or of yet unknown type. HS $2333+3927$

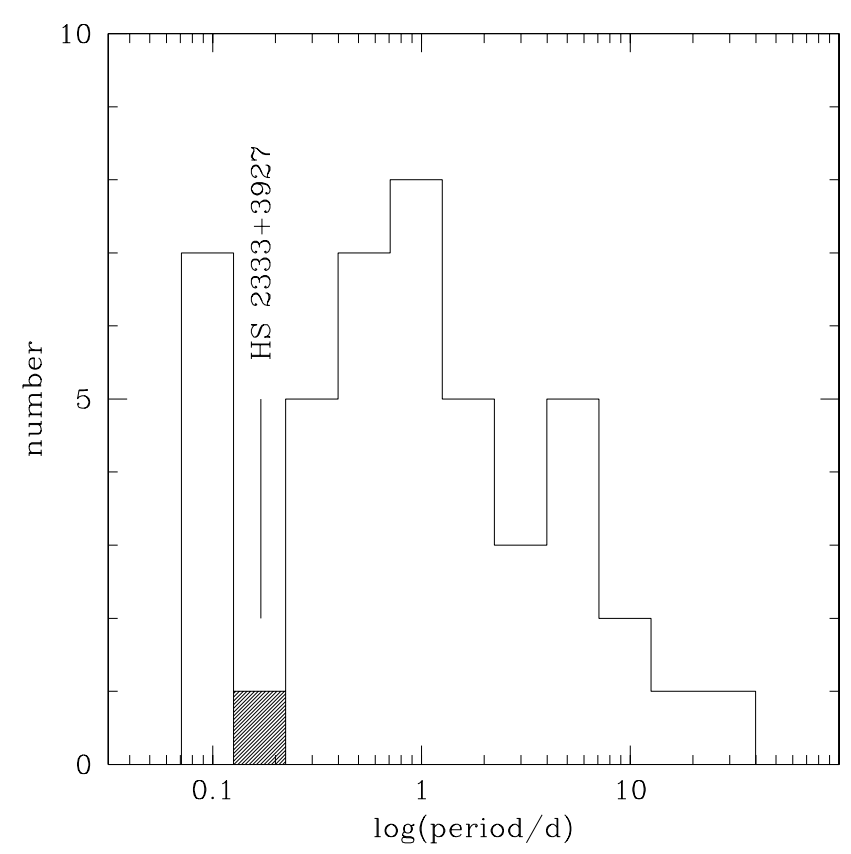

Fig. 11. Distribution of periods of known sdB binaries. HS $2333+3927$ (shaded) lies in the gap between the seven shortest period systems and the main bulk.

appears to link the seven shortest period systems to the bulk of objects.

Short period sdB binaries with main sequence companions, such as HS $2333+3927$, must have gone through a common envelope stage when the primary was near the tip of the red giant branch. Therefore they are important in order to understand the formation and evolution of sdB stars. These systems will evolve into cataclysmic variables when the orbital separation of the components shrinks. Therefore, these objects are also crucial to understand pre-CV evolution. While the four other known systems have periods between $1.7 \mathrm{~h}$ and $2.8 \mathrm{~h}$, i.e. below the CV period gap, the period ( $4.12 \mathrm{~h}$ ) of HS $2333+3927$ is above the $\mathrm{CV}$ period gap.

Acknowledgements. Financial support by the Deutsche Forschungsgemeinschaft for C. Karl (grant NA365/2-2), by the Bundesministerium für Bildung und Forschung (BMBF) for O. Cordes (grant 05AE2PDA/8), and by the Deutsches Zentrum für Luft- und Raumfahrt for M. Altmann (50 QD 0102) is gratefully acknowledged. Work on this project in Kiel was supported by grant FKZ 05 AE $2 \mathrm{GU} \mathrm{A} / 5$ from the BMBF and by financial support from the Christian-Albrechts-University, Kiel, Germany. This project has been supported by the European Commission through the "Access to Research Infrastructures Action" of the "Improving Human Potential Programme", awarded to the Instituto de Astrofisica de Canarias to fund European Astronomers' access to the European Northern Observatory, in the Canary Islands.

We thank the director of Calar Alto observatory, R. Gredel, for allocating some of his discretionary time at the $2.2 \mathrm{~m}$ telescope to our project and the director of the Isaac Newton Group, R. Rutten, for the discretionary time granted to R. $\varnothing$ on the JKT. The staff of Calar Alto observatory is thanked for taking the spectra as a service to us.

The German-Spanish Astronomical Center (DSAZ), Calar Alto, is operated by the Max-Planck-Institut für Astronomie Heidelberg jointly with the Spanish National Commission for Astronomy. 
The Jacobus Kapteyn Telescope is operated by the Isaac Newton Group and the Nordic Optical Telescope jointly by Denmark, Finland, Iceland, Norway, and Sweden. Both telescopes are in the Spanish Observatorio del Roque de los Muchachos of the Instituto de Astrofísica de Canarias on the island of La Palma. The data during the discovering run have been taken using ALFOSC, which is owned by the Instituto de Astrofisica de Andalucia (IAA) and operated at the Nordic Optical Telescope under agreement between IAA and the NBIfAFG of the Astronomical Observatory of Copenhagen.

\section{References}

Baraffe, I., \& Chabrier, G. 1996, ApJ, 445, 433

Bertin, E., \& Arnouts, S. 1996, A\&A, 117, 393

Billères, M., Fontaine, G., Brassard, P., \& Liebert, J. 2002, ApJ, 578, 515

Brassard, P., Fontaine, G., Billeres, M., et al. 2001, ApJ, 563, 1013

Brown, T. M., Ferguson, H. C., Davidsen, A. F., \& Dorman B. 1997, ApJ 482, 685

Brown, T. M., Bowers, C. W., Kimble, R. A., Sweigart, A. V., \& Ferguson, H. C. 2000a, ApJ, 532, 308

Brown, T. M., Bowers, C. W., Kimble, R. A., \& Ferguson, H. C. 2000b, ApJ, 529, L55

Charpinet, S. 2001, AN, 322, 387

Clemens, J. C., Reid, I. N., Gizis, J. E., \& O’Brien, M. S. 1998, ApJ, 496,352

Díaz-Cordovés, J., Claret, A., \& Giménez, A. 1995, A\&AS, 110, 329

O’Donoghue, D., Koen, C., Kilkenny, D., \& Stobie, R. S. 1999, in Proc. of the 11th European Workshop on White Dwarfs, ed. J.-E. Solheim, \& E. G. Meištas, ASP Conf. Ser., 169, 149

Dorman, B., Nelson, L. A., \& Chau, W. Y. 1989, ApJ, 342, 1003

Dorman, B., Rood, R. T., \& O'Connell, R. W. 1993, ApJ, 419, 596

Drechsel, H., Haas, S., Lorenz, R., \& Gayler, S. 1995, A\&A, 294, 723

Drechsel, H., Heber, U., Napiwotzki, R., et al. 2001, A\&A, 379, 893

Driebe, T., Schönberner, D., Blöcker, T., \& Herwig, F. 1998, A\&A, 339,123

Drilling, J. S., \& Landolt, A. U. 2000, in Allen's Astrophysical Quantities (4th edition), ed. A. N. Cox (New York: AIP Press, Springer)

Edelmann, H., Heber, U., Hagen, H.-J., et al. 2003, A\&A, 400, 939

Ferraro, F., Paltrinieri, B., Fusi Pecci, F., et al. 1997, ApJ, 484, L145

Hagen, H.-J., Groote, D., Engels, D., \& Reimers, D. 1995, A\&AS, 111,195

Han, Z., Podsiadlowski, P., Maxted, P. F. L., Marsh, T. R., \& Ivanova, N. 2002, MNRAS, 336, 449

Han, Z., Podsiadlowski, P., Maxted, P. F. L., \& Marsh, T. R. 2003, MNRAS, 341, 669

Heber, U. 1986, A\&A, 155, 33

Heber, U., Napiwotzki, R., \& Reid, I. N. 1997, A\&A, 323, 819

Heber, U., Edelmann, H., Lemke, M., Napiwotzki, R., \& Engels, D. 1999, PASPC, 169, 551

Heber, U. 2003, in White Dwarfs, ed. M. de Martino, R. Silvotti, J.-E. Solheim, \& R. Kalytis, NATO Sci. Ser. II, 105, 49
Heber, U., Edelmann, H., Lisker, T., \& Napiwotzki, R. 2003b, A\&A, 411, L477

Hilditch, R. W., Harries, T. J., \& Hill, G. 1996, MNRAS, 279, 1380

Hilditch, R. W., Kilkenny, D., Lynas-Gray, A. E., \& Hill, G. 2003, MNRAS, 344, 644

Kallrath, J., \& Linnell, A. P. 1987, ApJ, 313, 346

Kilkenny, D., O'Donoghue, D., Koen, C., \& van Wyk, F. 1998, MNRAS, 296, 329

Kwee, K. K., \& van Woerden, H. 1956, Bull. Astron. Inst. of Netherlands, 12, 327

Lucy, L. B. 1967, Z. Astrophys., 65, 89

Maxted, P. F. L., Heber, U., Marsh, T. R., \& North, R. C. 2001, MNRAS, 326, 1391

Maxted, P. F. L., Marsh, T. R., Heber, U., et al. 2002, MNRAS, 333, 231

Maxted, P. F. L., Marsh, T. R., \& Morales-Rueda, L. 2003, in White Dwarfs, ed. M. de Martino, R. Silvotti, J.-E. Solheim, \& R. Kalytis, NATO Sci. Ser. II, 105, 97

Morales-Rueda, L., Maxted, P. F. L., Marsh, T. R., North, R. C., \& Heber, U. 2003, MNRAS, 338, 752

Menzies, J. W., \& Marang, F. 1986, IAUS, 138, 305

Napiwotzki, R. 1997, A\&A, 322, 256

Napiwotzki, R., Green, P. J., \& Saffer, R. A. 1999, ApJ, 517, 399

Napiwotzki, R., Karl, C. A., Lisker, T., et al. 2004, in Extreme Horizontal Branch Stars and Related Objects, Kluwer, in press [arXiv: astro-ph/0401201]

Østensen, R. 2000, Time Resolved CCD Photometry, Ph.D. Thesis, University of Troms $\varnothing$, Norway

Østensen, R., Solheim, J.-E., Heber, U., et al. 2001a, A\&A, 368, 175

Østensen, R., Heber, U., Silvotti, R., et al. 2001b, A\&A, 378, 466

Rauch, T. 2000, A\&A, 356, 665

Rucinski, S. M. 1969, Acta Astr., 19, 245

Saffer, R. A., Bergeron, P., Koester, D., \& Liebert, J. 1994, ApJ, 432, 351

Saffer, R. A., Green, E. M., \& Bowers, T. P. 2001, in Proc. of the 12th European Workshop on White Dwarf Stars, ed. H. L. Shipman, J. L. Provencal, J. MacDonald, \& S. Goodchild (San Francisco: ASP), ASP Conf. Ser., 226, 408

Schuh, S., Dreizler, S., Deetjen, J.-L., Heber, U., \& Geckeler, R.-D. 1999, Baltic Astron., 9, 395

Shafter, A. W. 1992, in Proc. San Diego Conf. on Fundamental Parameters in Cataclysmic Variables (San Diego State University), ed. A. W. Shafter, 39

Vaz, L. P. R. 1985, Ap\&SS, 113, 349

Wade, R. A., \& Rucinski, S. M. 1985, A\&AS, 60, 471

Wilson, R. E., \& Devinney, E. J. 1971, ApJ, 166, 605

Wood, J. H., \& Saffer, R. 1999, MNRAS, 305, 82

Wood, J. H., Zhang, E.-H., \& Robinson, E. L. 1993, MNRAS, 261, 103

Zahn, J.-P. 1977, A\&A, 57, 383

von Zeipel, H. 1924, MNRAS, 84, 665 